\title{
Congenital Genetic Microcephaly: Clinical Diagnostic Approach
}

\author{
Silvia Marino ${ }^{1}$ Piero Pavone $^{1}$ Lidia Marino $^{1}$ Filippo Andrea Salvatore Rapisarda ${ }^{2}$ Raffaele Falsaperla ${ }^{1}$
}

\author{
${ }^{1}$ Department of Clinical and Experimental Medicine, Section of \\ Pediatrics and Child Neuropsychiatry - Pediatrics and Pediatric \\ Emergency, University Hospital "Policlinico-Vittorio Emanuele," \\ Catania, Italy \\ 2 Department of Maternal and Child - Gynecology and Obstetrics, \\ ARNAS Garibaldi-Nesima, Catania, Italy \\ J Pediatr Neurol 2020;18:131-134.
}

\begin{abstract}
Address for correspondence Silvia Marino, MD, Department of Clinical and Experimental Medicine, Section of Pediatrics and Child Neuropsychiatry, Pediatrics and Pediatric Emergency, University Hospital "Policlinico-Vittorio Emanuele"- Via Santa Sofia, 78 - 95100 Catania, Italy (e-mail: silvia_marino86@hotmail.it).
\end{abstract}

\begin{abstract}
Keywords

- microcephaly

- neonatology

- genetic

Microcephaly is an important neurological sign defined by a cranial circumference $<2$ standard deviations or $<3$ standard deviations in the severe form compared with ageand gender-matched children. Microcephaly is classified as congenital (prenatal) and postnatal. In general, microcephaly may result from an insult, including infections, metabolic diseases, cerebral malformations, and/or genetic syndromes that disturb early brain growth. Clinical history, the trajectory of the child's growth in terms of head circumference, and a detailed physical examination will often be suggestive of a diagnostic workup. Advances in neuroimaging and especially genetics have yielded improvements in understanding the causes of microcephaly, leading to new approaches in diagnosis, treatment, and prevention. The aim of the present study is to report the current practice for the diagnostic algorithm of genetic microcephaly, with prenatal onset.
\end{abstract}

\section{Introduction}

Microcephaly refers to a clinical condition characterized by a "head circumference that is $<2$ standard deviations (SDs) below the mean compared to age- and gender-matched population-based samples." ${ }^{1}$ Causes of microcephaly can be genetic syndromes, cerebral malformations, epileptic encephalopathies, infectious diseases (such as toxoplasmosis, others [syphilis, varicella-zoster, parvovirus B19], rubella, Cytomegalovirus, and herpes infections [TORCH] and Zika viruses). ${ }^{2-7}$ The correct diagnostic procedure allows for early multidisciplinary intervention, possible gene therapy, and microcephaly identification in future pregnancies. Unfortunately, this is not always possible due to the multiple causes of microcephaly. We have reviewed the literature concerning this topic and have proposed a potential diagnostic work-up for genetic microcephaly.

\section{Definition}

Microcephaly is a heterogeneous group of clinical conditions characterized by a cranial circumference length of $<2$ SDs based on age, gender, and ethnicity according to the curves of reference. ${ }^{8}$ For severe microcephaly, a head circumference of $<3$ SDs below the normal age- and gender-matched control is indicated. ${ }^{9-12}$ The measurement of the cranial circumference is part of a newborn's physical examination. The measurement must be performed as part of the routine clinical examination in the first 2 years of life. In an infant, it provides useful information concerning brain mass development. For the cranial measurements, it is necessary to use a flexible, nonstretchable measuring tape. A flexible millimeter tape, which is not extensible, must first pass over the front of the head above the superior orbital corresponding to the frontal sinus, laterally in a symmetric way, and in

Copyright $\odot 2020$ by Georg Thieme Verlag KG, Stuttgart · New York
DOI https://doi.org/ 10.1055/s-0039-1692970. ISSN $1304-2580$. 
the occipital region so as to measure the maximal circumference. This measurement should be noted from birth until the age of 2 years. ${ }^{2}$ The various measurements should be reported using the appropriate growth curves (World Health Organization-based curves or Intergrowth 21st are recommended $)^{8}$ for monitoring the trends over time. Normal circumferences are considered to be between the 3rd and the 97th percentile, but it will be important to monitor the increase in circumference longitudinally in doubtful cases. ${ }^{2}$

\section{Classification}

There is no standardized classification for microcephaly. General microcephaly is divided into congenital (prenatal or primary) or acquired (postnatal or secondary). Prenatal microcephaly includes a group of hereditary pathological conditions that follows Mendelian genetics and usually is not associated with other defects or specific genetic syndromes. Affected infants are usually identified at birth by a lower-than-average head circumference. In newborns, congenital microcephaly is present in utero and at birth, and in the acquired forms it manifests itself in the postneonatal ages. Moreover, primary form is characterized by a disorder of neuronal proliferation and a secondary form, characterized by acquired etiopathogenesis. In both cases, when the head circumference is below the age ranges, the causes may or may not be genetic. ${ }^{2}$

\section{Genetic Etiology}

The genetic causes of microcephaly are heterogeneous. Hundreds of microcephaly-associated syndromes have been described in the literature. It is possible to consult the Website, Online Mendelian Inheritance in Man, for an idea. Both homozygous and heterozygous genetic mutations have been recognized. Genetic tests, such as simple karyotyping, comparative genomic hybridization (CGH) arrays, whole exome, and, lastly, next-generation sequencing (NGS) can be used for this type of testing. Gene research must be addressed by clinical history and objective examination. At present, the NGS panel is able to identify genes, such as the abnormal spindle-like microcephaly-associated protein (ASPM), calcium/calmodulin-dependent serine protein kinase gene (identified in mental retardation and microcephaly with pontine and cerebellar hypoplasia), cyclin-dependent kinase, ${ }^{5}$ and ras-related protein rap-2 gene (identified in Seckel syndrome and microcephaly). ${ }^{2,13-15}$

\section{Microcephaly Primary Hereditary}

Autosomal recessive primary microcephaly (MCPH) is a rare heterogeneous genetic disorder of normal brain development characterized by a reduction in the head circumference of $<2$ SDs compared with the average for age, ethnicity, and intellectual disabilities. ${ }^{16,17}$ Microcephaly can be observed in the prenatal stage. Less frequent clinical signs are usually tonic/clonic seizures responsive to antiepileptic therapy, ${ }^{10,11}$ language delay, and hyperactivity. The incidence of MCPH is approximately 1 in 10,000 neonates. ${ }^{16,18}$ To date, 16 genes have been identified that underlie MCPH with mutations in the ASPM gene representing $50 \%$ of cases ${ }^{16-18}$ followed in frequency by WE-repeat-containing protein 62 mutations. ${ }^{19,20}$ These genetic mutations appear to be responsible for the reduction in the cortical brain neuron development during embryonic neurogenesis. Short stature is a classic feature of Seckel's syndrome, and it has also been reported in some people with mutations in the microcephalin (MCPH)1, 5, 6, 9, and 11 genes. ${ }^{21}$ Horizontal and prominent ears, arched palate, short toes, and inverted nipples can be noticed in individuals with MCPH2. ${ }^{21-25}$

\section{Diagnostic Workup}

Diagnosis and evaluation of microcephaly are essential to determine the cause, associated conditions, and genetic counseling for risk prevention, future pregnancies, and prognosis. A detailed history (travel in endemic area such as Zika virus and/or other) and a complete clinical examination are the first steps in the evaluation of a microcephaly program. In fact, more often Zika virus infection is found to be responsible for a congenital microcephaly, especially in South America and Asia. The Zika virus is responsible for a microcephaly associated with other abnormalities such as eye abnormalities, craniofacial disproportion, and some articular deformities and members, sometimes in the absence of microcephaly. ${ }^{26,27}$ Measuring the circumference of the head in parents and siblings is important because it can help diagnose familial microcephaly. The choice of laboratory outcomes is determined by anamnesis and the physical examination. It is useful to use neuroimaging in the presence of early fusion of the sutures, either cerebral ultrasound or even better, magnetic resonance imaging of the brain. The latter allows to identify the brain's structural abnormalities (for instance, lissencephaly, pachygyria, or polymicrogyria). An integral part of the workup of different diseases is genetic testing. Karyotyping and CGH array, which are now routinely performed in the case of newborns who have malformations, are used to compare the newborn to the older child in the setting of an intellectual disability. If karyotype and the CGH array are not useful in coming to a definitive diagnosis, group sequencing, whole exome, or whole genome testing may be used to allow for the identification of the underlying genetic abnormalities. The possibilities for genetic analyses have radically changed in the last decade, and microarray analyses have become the standard criterion. NGS methods are able to clarify the underlying cause in patients in whom the etiology of microcephaly is unknown (-Fig. 1). von der Hagen et al conducted a retrospective study of 680 children with microcephaly to evaluate the diagnostic approach to microcephaly in infancy and to identify the prevalence of various causes/ extent of the disease. Of these, the etiology was identified in only $59 \%$ of the samples, and of these, almost half showed a genetic cause identified by karyotyping, CGH array analysis, chromosomal breakage analysis, and sequencing of selected genes. The authors emphasize that this study was a retrospective study concerning children who presented to the center for intellectual disability and/or epilepsy, for which 


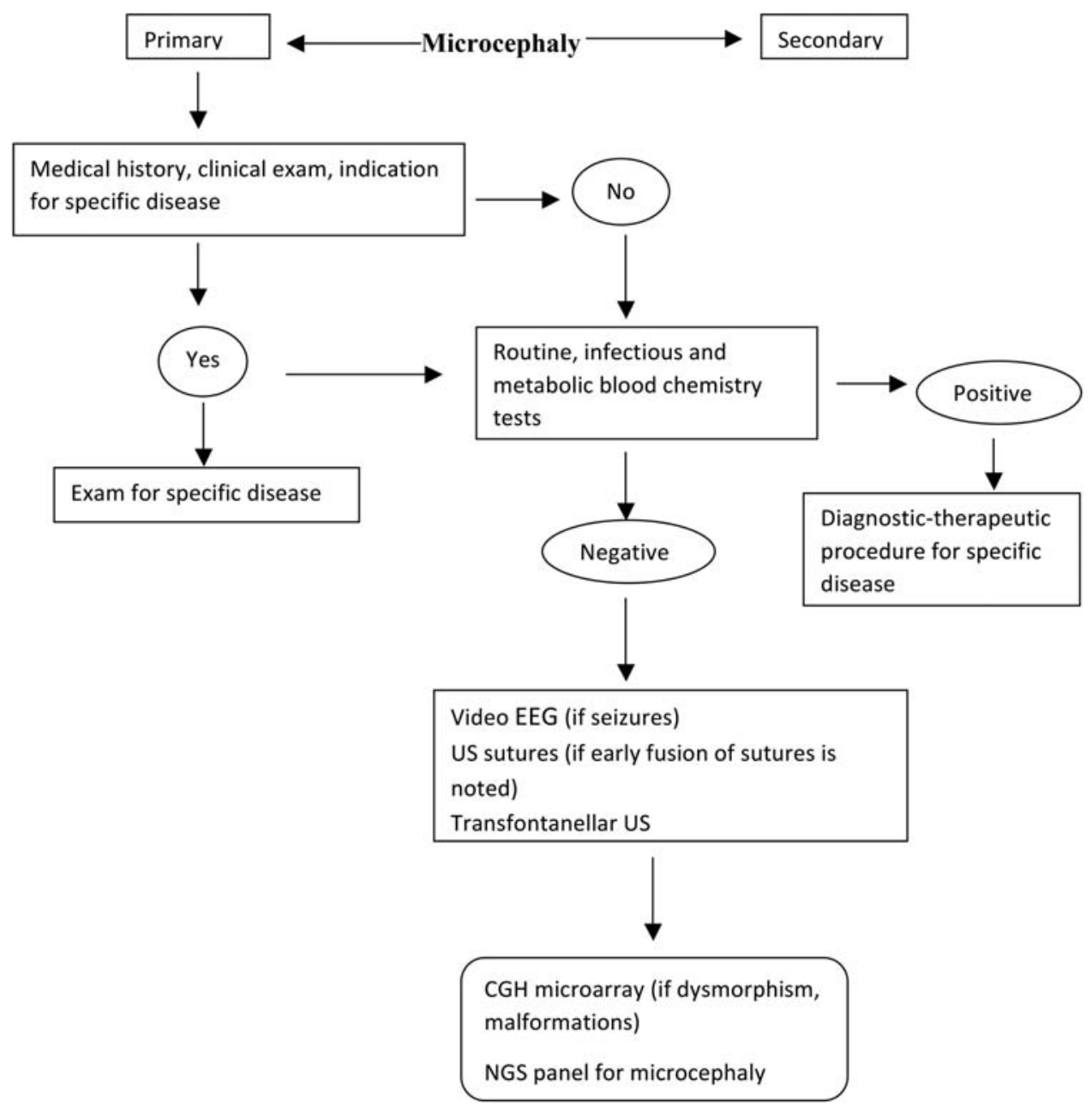

Fig. 1 Diagnostic algorithm for the evaluation of congenital genetic microcephaly. CGH, comparative genomic hybridization; EEG, electroencephalogram; NGS, next-generation sequencing; US, ultrasound.

the CGH array is now a routine test. ${ }^{2}$ However, further studies need to be performed to understand which children need to undergo genetic testing.

\section{Conclusion}

Microcephaly can be an isolated sign or, more often, a part of a constellation of signs of several rare genetic diseases. The NGS genetic panel test for microcephaly should be routinely included in doubtful cases to distinguish prenatal infections from other syndromes and/or genetic cause, as a test for future pregnancies (in family with other members with microcephaly) and, in the future to establish a potential correlation between genotype and phenotype. In the future, gene therapy could be a solution for genetic microcephaly. However, further studies are necessary to assess the value and effectiveness of this form of treatment.

\section{Ethical Approval}

The authors have no ethical conflicts to disclose.

Conflict of Interest

None declared.

\section{Acknowledgment}

We wish to thank the American Manuscript Editors (United States) for editing the manuscript.

\section{References}

1 Harris SR. Congenital idiopathic microcephaly in an infant: congruence of head size with developmental motor delay. Dev Neurorehabil 2013;16(02):129-132

2 von der Hagen M, Pivarcsi M, Liebe J, et al. Diagnostic approach to microcephaly in childhood: a two-center study and review of the literature. Dev Med Child Neurol 2014;56(08):732-741

3 Ashwal S, Michelson D, Plauwner L, et al. Quality Standards Subcommittee of the American Academy of Neurology and the Practice Committee of the Child Neurology Society. Practice parameter: evaluation of the child with microcephaly (an evidence-based review): report of the Quality Standards Committee of the American Academy of Neurology and the Practice Committee of the Child Neurology Society. Neurology 2009;73(11):887-897

4 Baxter PS, Rigby AS, Rotsaert MH, Wright I. Acquired microcephaly: causes, patterns, motor and IQ effects, and associated growth changes. Pediatrics 2009;124(02):590-595

5 Macfarlane DW, Boyd RD, Dodrill CB, Tufts E. Intrauterine rubella, head size, and intellect. Pediatrics 1975;55(06):797-801

6 Noyola DE, Demmler GJ, Nelson CT, et al; Houston Congenital CMV Longitudinal Study Group. Early predictors of neurodevelopmental 
outcome in symptomatic congenital cytomegalovirus infection. J Pediatr 2001;138(03):325-331

7 Faizan MI, Abdullah M, Ali S, Naqvi IH, Ahmed A, Parveen S. Zika virus-induced microcephaly and its possible molecular mechanism. Intervirology 2016;59(03):152-158

8 Villar J, Altman DG, Purwar M, et al; International Fetal and Newborn Growth Consortium for the 21st Century. The objectives, design and implementation of the INTERGROWTH-21st Project. BJOG 2013;120(Suppl 2):9-26

9 Leviton A, Holmes LB, Allred EN, Vargas J. Methodologic issues in epidemiologic studies of congenital microcephaly. Early Hum Dev 2002;69(1-2):91-105

10 Opitz JM, Holt MC. Microcephaly: general considerations and aids to nosology. J Craniofac Genet Dev Biol 1990;10(02):175-204

11 Abuelo D. Microcephaly syndromes. Semin Pediatr Neurol 2007; 14(03):118-127

12 Gilmore EC, Walsh CA. Genetic causes of microcephaly and lessons for neuronal development. Wiley Interdiscip Rev Dev Biol 2013;2(04):461-478

13 Pavone P, Praticò AD, Ruggieri M, Rizzo R, Falsaperla R. Resuming the obsolete term "small head": when microcephaly occurs without cognitive impairment. Neurol Sci 2017;38(09):1723-1725

14 Woods CG. Human microcephaly. Curr Opin Neurobiol 2004;14 (01):112-117

15 Leroy JG, Frías JL. Nonsyndromic microcephaly: an overview. Adv Pediatr 2005;52:261-293

16 Van Den Bosch J. Microcephaly in the Netherlands: a clinical and genetical study. Ann Hum Genet 1959;23(02):91-116

17 Jackson AP, McHale DP, Campbell DA, et al. Primary autosomal recessive microcephaly (MCPH1) maps to chromosome $8 \mathrm{p} 22$ pter. Am J Hum Genet 1998;63(02):541-546

18 Kleber de Oliveira W, Cortez-Escalante J, De Oliveira WT, et al. Increase in reported prevalence of microcephaly in infants born to women living in areas with confirmed Zika virus transmission during the first trimester of pregnancy - Brazil, 2015. MMWR Morb Mortal Wkly Rep 2016;65(09):242-247

19 Tunca Y, Vurucu S, Parma J, et al. Prenatal diagnosis of primary microcephaly in two consanguineous families by confrontation of morphometry with DNA data. Prenat Diagn 2006;26(05): 449-453

20 Kraemer N, Picker-Minh S, Abbasi AA, et al. Genetic causes of MCPH in consanguineous Pakistani families. Clin Genet 2016;89 (06):744-745

21 Bhat V, Girimaji SC, Mohan G, et al. Mutations in WDR62, encoding a centrosomal and nuclear protein, in Indian primary microcephaly families with cortical malformations. Clin Genet 2011;80(06):532-540

22 Barbelanne M, Tsang WY. Molecular and cellular basis of autosomal recessive primary microcephaly. BioMed Res Int 2014; 2014:547986

23 Mahmood S, Ahmad W, Hassan MJ. Autosomal recessive primary microcephaly (MCPH): clinical manifestations, genetic heterogeneity and mutation continuum. Orphanet J Rare Dis 2011;6:39

24 Kaindl AM, Passemard S, Kumar P, et al. Many roads lead to primary autosomal recessive microcephaly. Prog Neurobiol 2010; 90(03):363-383

25 Passemard S, Titomanlio L, Elmaleh M, et al. Expanding the clinical and neuroradiologic phenotype of primary microcephaly due to ASPM mutations. Neurology 2009;73(12):962-969

26 Del Campo M, Feitosa IM, Ribeiro EM, et al; Zika Embryopathy Task Force-Brazilian Society of Medical Genetics ZETF-SBGM. The phenotypic spectrum of congenital Zika syndrome. Am J Med Genet A 2017;173(04):841-857

27 França GVA, Pedi VD, Garcia MHO, Carmo GMID, Leal MB, Garcia LP. Congenital syndrome associated with Zika virus infection among live births in Brazil: a description of the distribution of reported and confirmed cases in 2015-2016 [in Portuguese]. Epidemiol Serv Saude 2018;27(02):e2017473 